\title{
Application and Challenges of Big Data and Cloud Computing in Power Industry
}

\author{
Jinsong Zhang* \\ School of Management, Tianjin University of Technology, Tianjin, China. \\ *806338091@qq.com
}

\begin{abstract}
The rapid development of informatization has led to an exponential explosion in the amount of information. As the basic energy support system for the world's second largest economy, China has also entered the era of power big data. At the same time, in view of the development status of China's power industry, high requirements are placed on management and data servers. Big data technology and cloud computing systems are used in the electric power industry by virtue of their own advantages. On the basis of introducing the concepts of big data and cloud computing, various applications of big data and cloud computing in power industry and the challenges they faced are analyzed. At the same time, the direction of future development of big data and cloud computing in the power industry is further expanded.
\end{abstract}

Keywords: big data; cloud computing; data servers.; support system.

\section{Introduction}

According to the forecast of Global Data Corporation (IDC), the current annual growth rate of new data is as high as $50 \%$. In short, the number of data applications doubled every two years, which means that the era of big data has come. With the explosive growth of data, there have been tremendous changes in all walks of life around the world. As the world's second-largest basic energy system, China's power industry has also entered the era of big data, with all sectors and links (grid, power generation, transmission, distribution, electricity, etc.) having entered the era of power big data. However, given the wide scope of management involved in China's power industry, it includes not only actual production, specific sales, production scheduling and corporate finance, but also production safety, management plans, regulations, and many other aspects. In the era of big data, higher requirements are put forward to industrial computing server and data server computing capabilities. Among them, "big data technology" and "cloud computing" have been widely used in the power industry due to their advantages of high security, high reliability, and high timeliness in data processing and management classification. Therefore, this paper elaborates the concept of big data and cloud computing, and systematically analyzes the application of big data and cloud computing in power industry. It is hoped that the paper is of help to expand the development scope of big data and cloud computing in power industry.

\section{Overview of Big Data}

"Big Data" means that the quantity of material or data cannot be analyzed with the mainstream software at the current stage, and collection, management, analysis, and summary as the data information which helps managers to make decision within the prescribed time. The characteristics of big data exhibit are mainly largeness, diversity, high-speed and value. "Big data technology" is a means that can effectively collect, process, analyze, and organize massive data along with the information industry and computer technology, and presents a high level of decision-making, discovery, and deployment capabilities. "Big data technology" is not only the scientific and technological product of the development of science and technology, but also the wisdom of mankind in transforming the nature. At the current stage, the "Hadoop Distributed" system infrastructure is primarily created by the Apache Foundation and is the widest used infrastructure for processing big data. 


\section{Overview of Cloud Computing}

Under the background of the demand for massive data computing and data storage by the Internet companies, cloud computing has emerged as a new distributed computing model. Cloud computing aggregates massive, distributed and heterogeneous resources to meet customers' requirements for high scalability, high performance, and high availability for resource storage and data calculation. At the same time, cloud computing adopts virtualization technologies and other means to meet various customers' needs as much as possible, prevents resource waste and vicious competition, and further enhances the efficiency of resource utilization. In addition, cloud computing also has a high level ability to balance horizontal scaling and dynamic load. In other words, cloud computing supports adding new nodes to the data center during the operation process, and the system will automatically transfer some of the loads to the new ones. It also ensures that the load between the nodes will not be unbalanced, and further enhances the overall service carrying capacity of the power system. In cloud computing, resources are primarily arranged in a "data center" as a basic form. Among them, the data center includes thousands of nodes, and these nodes use high-speed networks to achieve mutual contact and provide customers with data computing and resource storage services.

\section{Big Data and Cloud Computing Applications in Electric Power Industry}

\subsection{Application of Power Data Prediction in Different Links}

The application of "big data technology" in power industry mainly aims at accurately predicting power data in different links, such as power generation, power distribution, transmission, etc., and then conducting targeted analysis of the data, and finally presenting it in an intuitive and visual data format in front of people. Taking the application of "big data technology" in forecasting power system load as an example, power load forecasting is a very crucial component in power management and distribution management systems, and the core of the work is mainly based on collecting a large amount of historical data. A scientific and reasonable data prediction model was established and many practical researches were carried out to finally explore the trends of power load changes. "Big data technology" is used in the main steps of forecasting power system load: collect and select a certain amount of historical power load data; preprocess power load data and sort out historical data; set up a scientific power load forecasting model; select the correct algorithm; implement electricity load forecasting. Power "big data technology" meets the rapid growth of power data, professional work, and improving the development of the power industry and the development of the service economy.

\subsection{Application of Managing Power Energy}

Applying "big data technology" to power energy management not only contributes to effective clean energy, but also helps to effectively control energy consumption. This measure is of crucial importance in the construction of modernization, technicalization, and intellectualization in power grid engineering projects and maintenance of the normal, stable, and safe operation of the power grid. Taking Beijing Dongfang Zhongke Integrated Co., Ltd. as an example, the company provides power products sales, power metering and calibration, and post-maintenance power maintenance services to customers. It has applied "big data technology" to power energy management, such as collecting, analyzing and testing power signal sources and data in the process of locomotives, electronic measurement industry, analysis of data in production links, new power applications, and other aspects.

\subsection{Application of Mining Power Data}

Data mining, also known as knowledge discovery, is a technique that finds its laws from a large amount of data by analyzing each data. The use of big data can analyze the business, process it into useful data, and then fully control the business. The application of "big data technology" in data mining of power industry is mainly to find out changes of power data. On one hand, it helps to summarize and analyze related power business conditions, and on the other hand, it helps to explore the application of high power data and ultimately enables power companies to effectively control 
related power businesses. In addition, with the rapid development of computer technology and Internet technology, power data shows an explosive growth trend, which further enhances the difficulty of mining power data. The application of "big data technology" in the mining of power industry data includes the following major steps: decomposition of power business and data; backup of power data; establishment of a scientific power mining data model; data evaluation and deployment.

The MapReduce algorithm architecture required for large data acquisition calculations is mapped first to a small computational unit and then all the calculation results are integrated. Some of the previous data mining techniques may not be easily embedded in the MapReduce framework. Some algorithms need to be adjusted before they can be used. Therefore, calculations performed on a single computer still require the use of some data mining techniques. Big data focuses on complexity of the data and is less accurate; analyzes comprehensive data rather than sampled individuals; and focuses on analyzing the relevance of data rather than causality.

\subsection{Application of Data Calculation}

Applying "cloud computing" to power industry system, on one hand, can automatically convert the resources to be stored according to the requirements of actual applications; and on the other hand, migrate the running nodes of the power grid and the single operation to the cloud of the computer. In the calculation system, all the data is finally processed by the "cloud". The use of "cloud computing" in power industry system can not only obtain a strong level of data calculation and resource storage, but also greatly strengthen the overall calculation level of power industry system.

In 2013, Jiangsu Provincial Electric Power Company took the lead in the construction of a big data intelligent analysis system. It used Hadoop distributed batch processing technology to establish a multi-dimensional data analysis model including electricity, power load, geographic information, meteorological data, and developed data. The visual display interface of the analysis results shows that the application of power to the economy, electricity to people's livelihood, and the user's electricity consumption behavior analysis are initially implemented.

\subsection{Application of Hierarchical Management}

The cloud computing hierarchical management in the power industry system mainly uses power grid to set up the "main cloud" and "sub-cloud" to carry out resource allocation and permission setting. Under normal circumstances, a certain level of cloud computing usually obtains the corresponding resources from the upper sub-cloud. Under emergency, emergency dispatch can be used to obtain other different levels of resources. In power system cloud computing, if there are multiple task requests, the task of assignment and submission mechanism will be generated. This approach mainly prevents the information from being blocked and insufficient resources. The application of cloud computing in power industry system is mainly based on the classification of power grid level and the implementation of task-level management. This is mainly because power industry has a huge system structure and a wide range of distribution. When all requests are submitted to cloud computing center, information jams will surely occur. Only by adopting task-level management can we effectively avoid them. Hierarchical and clear hierarchy management also adopts the method of level setting to further implement the "main cloud" and "sub-cloud" access and resource allocation, and finally form a scientific and rationalized cloud computing system for power industry.

\section{Introducing Big Data and Cloud Computing Challenges}

\subsection{Grid Data Infrastructure Needs Improvement}

For big data technologies and cloud computing systems, the quality of data and the strength of data management and control directly affect the accuracy and real-time performance of data mining analysis. The essence of big data technology and cloud computing system is to mine knowledge and improve data value by associating complex data. At present, there is a lack of sharing mechanism for grid data, poor communication and low data integration. With the continuous deepening of power grid information, data information between different business systems, especially between different 
departments, cannot be shared, affecting the operational efficiency of the enterprise. It is necessary to unify and integrate system models and data specifications. The power grid information network transmission capability and data storage, processing, and switching capabilities cannot meet the requirements. The grid data infrastructure needs to be constructed and improved.

\subsection{Information Security}

The current grid information platform lacks security defense capabilities and information security faces challenges. The geographical coverage of the power grid is extremely wide. The construction of the security protection system for the local information platform is unbalanced and the level of information security is inconsistent. The security protection system for the information platform in a particularly remote area has not yet been fully established and the security needs to be improved. The security protection measures and key protection measures for the power industry information platform also need to be further strengthened, from the current passive defense to multi-level and active defense.

\section{Conclusion}

To sum up, as a very important basic energy industry in China, how can power industry better apply "big data technology" and "cloud computing" to power industry is of crucial importance for future stable social development and stable development of the country and the stable development of power industry. Therefore, systematically analyzing the application and challenges of "big data technology" and "cloud computing" in power industry and exploring power big data information in power industry, will help power industry to continuously expand its business scope and better serve its customers. The ultimate realization of the long-term and harmonious development of the power industry plays a very important role.

\section{References}

[1]. JIN H T. Big data in power system [J]. Electronic Technology \&Software Engineering, 2017(6): 161.

[2]. HUANG L, TAO R. Power computing technology based on cloud computing and its application [J]. Digital Technology \&Applications, 2017(2): 117.

[3]. QI W K. Application of big data and visualization platform in electric power enterprises [J]. Electronic World, 2016(24): 138, 168.

[4]. YUAN H. Cloud computing research in the application of power industry [J]. Electronic Test, 2016(13): 108-109.

[5]. CAO W C. Application of big data platform in power system [J]. Jiangsu Science and Technology Information, 2016(29): 53-56, 71. 